\title{
ARTíCULO
}

\section{Modelo hidrodinámico en el Sistema Lagunar Navachiste, Guasave, Sinaloa, México}

\author{
Hydrodynamic model in the Lagoon System of \\ Navachiste, Guasave, Sinaloa, México

\section{Fernando de J. Sánchez-Lindoro ${ }^{1 *}$, Ángel R. Jiménez-Illescas², T. Leticia Espinosa-Carreón ${ }^{1}$ y Maclovio Obeso-Nieblas ${ }^{2}$}

\begin{abstract}
${ }^{1}$ Instituto Politécnico Nacional, Centro Interdisciplinario de Investigación para el Desarrollo Integral Regional, Blvd. Juan de Dios Bátiz Paredes \# 250, Col. San Joachin, Guasave, CP 81101, Sinaloa, México. *autor de correspondencia: fernando.sanchez.lindoro@gmail.com

${ }^{2}$ Instituto Politécnico Nacional, Centro Interdisciplinario de Ciencias Marinas, Ave. Inst. Politécnico Nacional S/N, Col. Playa Palo de Santa Rita, La Paz, B.C.S. 23090, México
\end{abstract}

\begin{abstract}
The Navachiste Lagoon System is connected to the Gulf of California (GC) via 3 tidal channels. The system has an area of approximately $301 \mathrm{~km}^{2}$, with an average depth of $2.5 \mathrm{~m}$ and an average tide height of $1.5 \mathrm{~m}$. In order to simulate tidal currents and the effect of wind, a numerical hydrodynamic model was applied, integrated in the vertical and in finite differences; to calibrate the model, lagrangean measurements were made and compared with simulated currents in the tidal channels of the system. The model simulates the hydrodynamic behavior of the lagoon system and has a correlation higher than $85 \%$ with the trajectories traced by the drift bodies and with the maximum Eulerian velocities measured in the internal channels and in the mouths of the system.
\end{abstract}

Key words: Tidal currents, coastal lagoons, flow simulation, hydrodynamic numerical model, San Ignacio-Navachiste-Macapule Lagoon System

\begin{abstract}
Resumen.- El Sistema Lagunar Navachiste se encuentra conectado con el Golfo de California (GC) a través de 3 canales de marea. El sistema cuenta con un área de $301 \mathrm{~km}^{2}$ aproximadamente, con una profundidad promedio de $2,5 \mathrm{~m}$ y una altura de mareas promedio de $1,5 \mathrm{~m}$. Para simular las corrientes de mareas y el efecto del viento se aplicó un modelo hidrodinámico numérico barótropico, integrado en la vertical y en diferencias finitas; para calibrar el modelo se hicieron mediciones lagrangeanas y se compararon con las corrientes simuladas en los canales de marea del sistema. El modelo simula el comportamiento hidrodinámico del sistema lagunar y presenta una correlación superior al $85 \%$ con las trayectorias trazadas por los cuerpos de deriva y con las velocidades máximas eulerianas medidas en los canales internos y en las bocas del sistema.
\end{abstract}

Palabras clave: Corrientes de marea, lagunas costeras, simulación de corrientes, modelo hidrodinámico numérico, Sistema Lagunar San Ignacio - Navachiste - Macapule

\section{INTRODUCCIÓN}

Las zonas costeras se caracterizan por una alta productividad y una alta biodiversidad, presentan muchas ventajas para el asentamiento de comunidades y para el desarrollo de actividades de producción alimenticia, como acuacultura y pesca, sin embargo, en los últimos años, el incremento poblacional en la región norte del estado de Sinaloa y el desarrollo de una agricultura y acuacultura altamente tecnificadas, con más de 200,000 Ha de cultivo, ha generado un importante impacto en los sistemas costeros, causando efectos como eutrofización, decremento en la calidad del agua, proliferaciones microalgales nocivas (Cifuentes et al. 1972, Millán-Núñez et al. 1981, Flores-Verdugo et al. 1993, Bustillos-Guzmán et al. 1995, Escobedo-Urías et al. 2007 y Espinosa-Carreón \& Valdez-Holguín 2007).
El Sistema Lagunar Navachiste, también conocido como Sistema Lagunar San Ignacio-Navachiste-Macapule se localiza al sureste del Golfo de California, es uno de los cuerpos costeros más importantes del estado de Sinaloa, cuenta con una importante complejidad hidrodinámica, así como una amplia actividad biológica y pesquera, es por ello, una región de gran interés científico y económico para el norte del estado de Sinaloa y México. La conexión con el golfo es continua y muy dinámica, ocurre a través de 4 canales de variación de mareas.

El Sistema Lagunar Navachiste, se encuentra influido principalmente por 2 patrones de viento: 1) Los vientos del sur y sureste, que ocurren al final de la primavera, persisten en verano y hasta el inicio del otoño, 2) los vientos del norte y 
noroeste alisios, que son dominantes a finales del otoño, invierno y principios de primavera, son fuertes y persistentes. Este patrón de vientos se debe principalmente a un carácter monzónico regional (Obeso-Nieblas et al. 1993, Jiménez-Illescas 1996, Obeso Nieblas et al. 1999, Obeso-Nieblas et al. 2012). Roden (1972), determinó que los campos de viento en el Golfo de California (GC), están principalmente influidos por el desplazamiento atmosférico del centro de alta presión del Pacífico y el centro de baja presión del Desierto de Sonora. En el GC, durante el invierno la intensidad de los campos de viento es alta $\left(8-12 \mathrm{~m} \mathrm{~s}^{-1}\right)$ provenientes del noreste, mientras, que en verano los vientos predominantes son del sureste $\left(5 \mathrm{~m} \mathrm{~s}^{-1}\right)$ (Douglas et al.1993). Según Molina-Cruz (1984), Salas (1996), y Marinone \& Lavín (1997), en verano los vientos dominantes del sureste son generados por el sistema de baja presión en la porción noreste del Golfo de California (Roden 1958), desplazando aire cálido y húmedo sobre el golfo.

El patrón de circulación del viento aunado a la profundidad promedio de este sistema lagunar menor a 2,5 m de acuerdo a las mediciones batimétricas realizadas para esta investigación produce un tiempo de retención significativo. El recambio de agua en la laguna Navachiste, Sinaloa, de acuerdo al cálculo realizado por balance de sal y agua del sistema fue de 16 días aproximadamente, mientras que para la laguna de Macapule fue de 2,2 días (Escobedo-Urías et al. 2007).

La zona marino-costera del complejo insular Sistema Lagunar Navachiste, se localiza en una zona de alta productividad fitoplanctónica, en la región sur del Golfo de California, influida por ser además zona de confluencia de aguas costeras sub-superficial fría y caliente superficial, rica en nutrientes (Espinosa-Carreón \& Valdez Holguín 2007).

Las islas del Sistema Lagunar Navachiste, son reconocidas como un Área Natural Protegida y alberga especies de animales y plantas sujetas a la conservación (CONANP 2010) ${ }^{1}$, decreto del 2 de agosto de 1978 por el que se establece una zona de refugio de aves migratorias y de la fauna silvestre, en las islas que se relacionan, situadas en el Golfo de California, México), además, el sistema recibe las escorrentías de los sistemas agrícola y acuícola, así como las descargas domésticas (con un volumen de $\left.1,48 \mathrm{~m}^{3} \mathrm{~s}^{-1}\right)$, sin tratamiento alguno, las cuales causan eutrofización del sistema, siendo éste un factor determinante en la alta productividad primaria y, en la dominancia de especies tolerantes a condiciones nutricionales alteradas por factores antropogénicos, tal como proliferaciones microalgales nocivas (Martínez-López et al. 2006, Poot-
Delgado 2006, Escobedo-Urías 2007, Martínez-López et al. 2007). Los escurrimientos tanto de los excedentes de riego como del agua de lluvia encauzada a través de los sistemas de drenaje varían estacionalmente en diferentes zonas. Los mayores gastos obtenidos para el Sistema Lagunar Navachiste, son en invierno y muestran el impacto de las prácticas agrícolas, debido a que coinciden los riegos para los cultivos de otoño-invierno y los de preparación para primavera-verano (De la Lanza-Espino \& Cáceres-Martínez 1994).

Por otro lado, los modelos numéricos cubren desde conceptos e hipótesis expresados en forma gráfica o descriptiva hasta formulaciones matemáticas complejas del comportamiento dinámico de los sistemas fisiológicos, así comolas predicciones de algunos fenómenos naturales mediante la simulación computacional. Una de las partes más importantes que se consideran en la construcción y aplicación de modelos numéricos matemáticos en sistemas costeros es entre otras cosas, alcanzar una mejor compresión del comportamiento de dichos sistemas, formulando cuantitativamente los fenómenos y la predicción del comportamiento del sistema sobre la base de pocos parámetros (Obeso-Nieblas 1987, Obeso-Nieblas et al. 1993, Jiménez et al. 1994, Jiménez-Illescas et al. 1997, Dworak 2005).

En el presente trabajo se realizó un estudio de la descripción hidrodinámica, mediante la implementación de un modelo numérico de diferencias finitas en el Sistema Lagunar Navachiste. Los resultados de esta investigación contribuirán en describir, determinar y explicar la dinámica del Sistema Lagunar Navachiste, Guasave, Sinaloa, México.

\section{MATERIALES Y MÉTODOS}

\section{Área DE ESTUdio}

El Sistema Lagunar Navachiste y su zona marina adyacente $\left(24,55^{\circ}-25,35^{\circ} \mathrm{N}, 108,3^{\circ}-109,03^{\circ} \mathrm{W}\right)$ se localizan al norte del Estado de Sinaloa, México (Fig. 1). Se ubica a $40 \mathrm{~km}$ al sur de la Ciudad de Guasave, Sinaloa, México (CONANP 2010)ํ․

El complejo incluye 3 lagunas: Laguna San Ignacio con un área aproximada de $49 \mathrm{~km}^{2}$, Laguna Navachiste con $206 \mathrm{~km}^{2}$ y Laguna Macapule con 46 km² (Escobedo-Urías et al. 1999).

La profundidad promedio del sistema lagunar es $2,5 \mathrm{~m}$, las zonas de mayor profundidad están localizadas en los canales internos y en las 3 conexiones con el GC: Boca de Ajoro, une Laguna San Ignacio y la parte norte de Laguna Navachiste con el GC, cuenta con un ancho de $2 \mathrm{~km}$ y una profundidad

${ }^{1}$ CONANP. 2010. Comisión Nacional de Áreas Naturales Protegidas. Decreto 2 de agosto de 1978 por el que se establece una zona de refugio de aves migratorias y de la fauna silvestre, en las islas que se relacionan, situadas en el Golfo de California, México.<http:/ /www.conanp.gob.mx/sig/decretos/apff/Islasgolfo.pdf> 


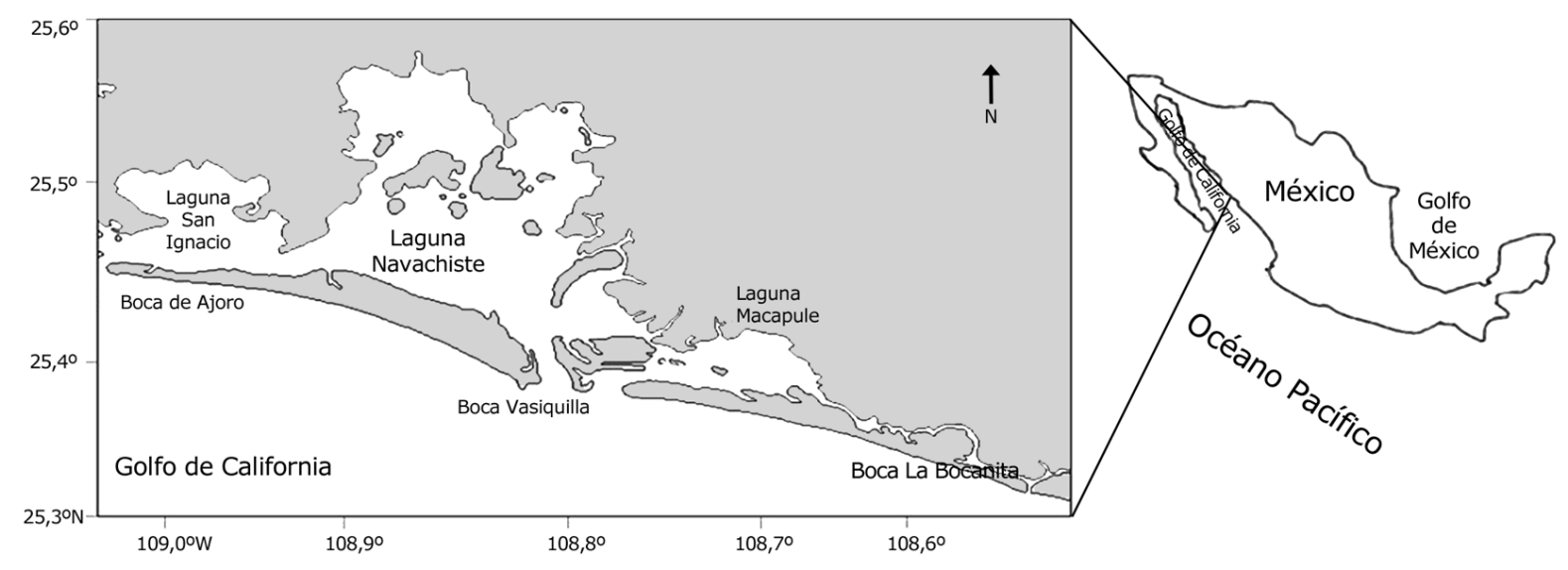

Figura 1. Localización del área de estudio del Sistema Lagunar Navachiste, Sinaloa, México / Location of the study area of the Navachiste Lagoon System, Sinaloa, Mexico

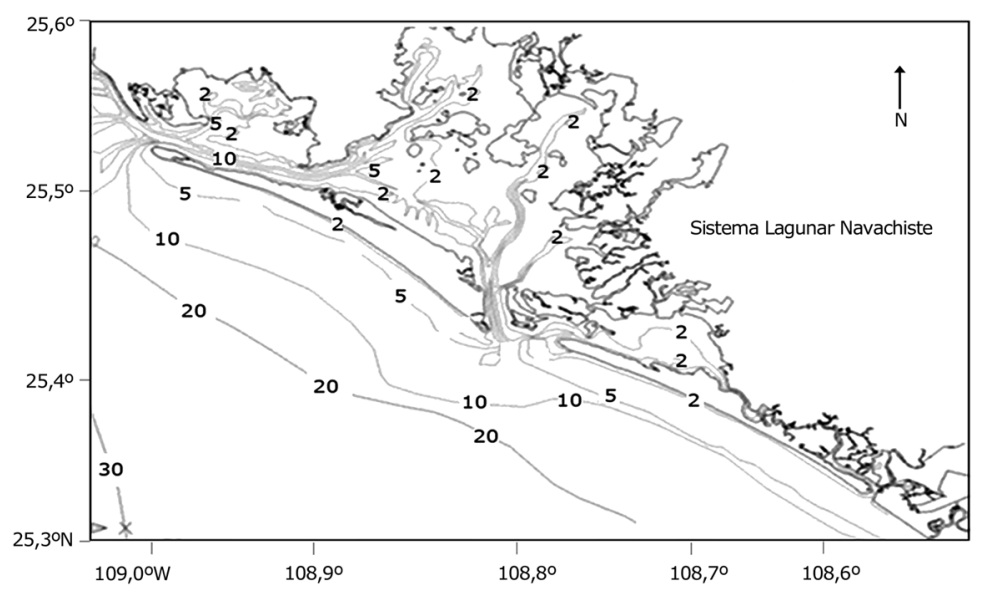

Figura 2. Batimetría de la región correspondiente al Sistema Lagunar Navachiste, y su región oceánica adyacente (profundidad en metros) / Bathymetry of the region corresponding to the Navachiste Lagoon System and its adjacent oceanic region (depth in meters)

aproximada de 9,5 m; Boca Vasiquilla conecta Laguna Navachiste con el GC, con un ancho 1,5 km y una profundidad 11,0 m; Canal de Macapule une el norte de la Laguna Macapule con el GC alcanzando una profundidad de 9,0 $\mathrm{m}$ y la Boca La Bocanita, tiene $8,0 \mathrm{~m}$ de profundidad y enlaza la parte sur de la Laguna Macapule con el GC (Magaña-Álvarez 2004, Escobedo-Urías et al. 2007, Zayas-Esquer 2010) (Fig. 2).

Las mareas son tipo mixto predominantemente semidiurno, 2 pleamares y 2 bajamares cada $24,84 \mathrm{~h}$, y en ocasiones presenta una marea diurna (una pleamar y una bajamar en 24,84 h) en mareas muertas. Cabe mencionar que siempre la pleamar superior es seguida por la bajamar inferior, causando que el reflujo mayor sea muy intenso, que es el principal factor que mantienen abiertos los canales de las bocas del sistema, evitando que el transporte de sedimentos desde el litoral se realice, ya que no hay otros aportes permanentes de ríos (sólo cuando hay lluvias extraordinarias) (Fig. 3).

Según García (1973), el sistema lagunar se encuentra en la franja de transición entre los climas semi-seco y muy cálido. El promedio de precipitación es de 365 a $450 \mathrm{~mm}$ por año, pero un tercio de las lluvias se presentan en agosto y septiembre. La evaporación anual es cercana a los $2.000 \mathrm{~mm}$ por año, con máximo de $2.498 \mathrm{~mm}$ por año y un mínimo de $1.358 \mathrm{~mm}$ por año. La temperatura ambiente promedio anual es de $23,5^{\circ} \mathrm{C}$. La producción pesquera del sistema lagunar se ha estimado en aproximadamente 500 ton anuales de camarón y 1.150 ton anuales de escama (Escobedo-Urías et al. 1999). 


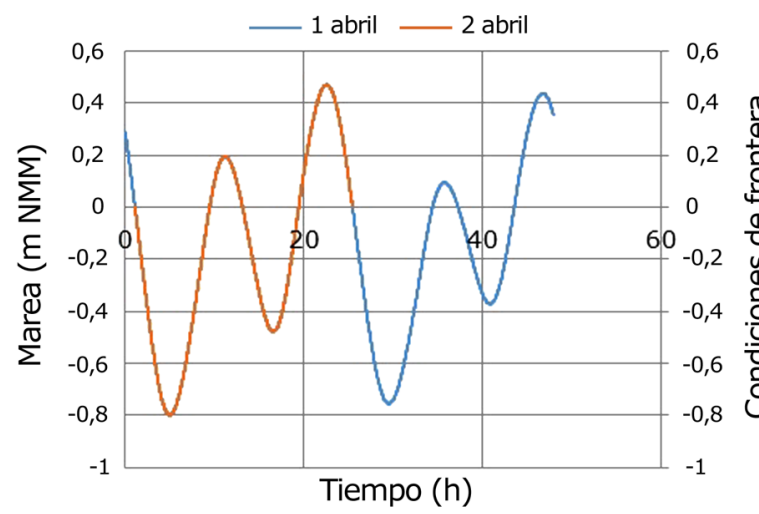

Figura 3. Oscilación de la marea en el Sistema Lagunar Navachiste del 1 y 2 de abril 2014 / Tidal oscillation in the Navachiste Lagoon System on 1 and 2 April 2014

Se utilizó un modelo bidimensional hidrodinámico numérico para predecir las corrientes inducidas por la marea y el esfuerzo producido por un viento uniforme (Gaviño \& Fernández 1987). Este modelo verticalmente integrado resuelve las ecuaciones de momento y continuidad. En el término de forzamiento por marea se incluyeron los armónicos componentes más representativos. En el forzamiento por viento se usaron vientos constantes del noroeste $5 \mathrm{~m} \mathrm{~s}^{-1}$.

Se utilizaron ecuaciones diferenciales parciales:

Cambio local, cambio advectivo, gradiente de presión, factor de Coriolis, Fricción; Viento-Fondo y Fricción Turbulenta.

en la dirección $\mathrm{x}$ :

$$
\begin{gathered}
\frac{\partial \bar{u}}{\partial t}+u \frac{\partial \bar{u}}{\partial x}+v \frac{\partial \bar{u}}{\partial y}+w \frac{\partial \bar{u}}{\partial z}=-g \frac{\partial \eta}{\partial x}+f \bar{v}+ \\
\frac{\tau_{s x}-\tau_{f x}}{\rho(h+\eta)}+A_{H} \nabla_{H}^{2}(\bar{u} h)
\end{gathered}
$$

en la dirección y:

$$
\begin{gathered}
\frac{\partial \bar{v}}{\partial t}+u \frac{\partial \bar{v}}{\partial x}+v \frac{\partial \bar{v}}{\partial y}+w \frac{\partial \bar{v}}{\partial z}=-g \frac{\partial \eta}{\partial y}-f \bar{v}+ \\
\frac{\tau_{s y}-\tau_{f y}}{\rho(h+\eta)}+A_{H} \nabla_{H}^{2}(\bar{v} h)
\end{gathered}
$$

donde $\tau_{s x}$ y $\tau_{s y}$ son las componentes del esfuerzo en la superficie, $\tau_{f x}$ y $\tau_{f y}$ son las componentes del esfuerzo en el fondo. El esfuerzo superficial es debido principalmente a la fricción del viento, mientras que la fricción en el fondo se debe a que el agua encuentra otro medio de diferente densidad.
Tabla 1. Símbolos de la ecuación de momento y continuidad / Symbols of the momentum and continuity equation

\begin{tabular}{ll}
\hline Símbolos & \\
\hline$(\mathrm{x}, \mathrm{y})$ & Coordenadas cartesianas \\
$\mathrm{U} y \mathrm{y}$ & $\begin{array}{l}\text { Transporte verticalmente integrado en la dirección } x \text { e } y \\
\text { respectivamente }\end{array}$ \\
$\mathrm{f}$ & Parámetro de Coriolis $=2 \mathrm{w}$ sen (latitud) \\
$\mathrm{w}$ & Velocidad angular de la tierra \\
$\mathrm{g}$ & Aceleración debida a la gravedad \\
$\mathrm{AH}$ & Coeficiente horizontal de viscosidad \\
$\mathrm{t}$ & Tiempo \\
$\mathrm{R}$ & Coeficiente de fricción en el fondo \\
$\mathrm{Hz}$ & Profundidad de la malla en los cuales se calcularán los \\
& transportes U y V \\
$\mathrm{H}$ & Profundidad instantánea del mar (H= Hz $+\mathrm{Z})$ \\
$\mathrm{t}_{\mathrm{b}}$ & Esfuerzo en el fondo \\
$\mathrm{t}_{\mathrm{w}}$ & Esfuerzo del viento \\
$\mathrm{r}_{\mathrm{w}}$ & Densidad del agua de mar \\
$\mathrm{r}_{\mathrm{a}}$ & Densidad del aire \\
$\mathrm{W}$ & Velocidad del viento \\
$\mathrm{V}$ & Velocidad promedio en la vertical \\
$\mathrm{C}_{\mathrm{b}}$ & Coeficiente de fricción para el esfuerzo en el fondo \\
$\mathrm{C}_{\mathrm{w}}$ & Coeficiente de fricción para el esfuerzo del viento \\
$\mathrm{Z}(\mathrm{x}, \mathrm{y}, \mathrm{t})$ & Variación de la profundidad del mar \\
$\mathrm{A}_{\mathrm{i}}(\mathrm{x}, \mathrm{y})$ & Amplitud de las constituyentes de la marea \\
$\mathrm{a}_{\mathrm{i}}(\mathrm{x}, \mathrm{y})$ & Fases de las constituyentes de la marea \\
$\mathrm{w}_{\mathrm{i}}$ & Velocidad angular de las constituyentes de la marea \\
$\eta$ & Nivel de la Superficie Libre (interfase agua-aire) \\
\hline &
\end{tabular}

Las ecuaciones anteriores se resolvieron numéricamente para encontrar el valor de las incógnitas $u, v, h$ que representan las componentes de la velocidad en dirección $x$, en dirección $y, y$ $\eta$ la elevación de la superficie libre.

$$
\partial_{x} U+\partial_{y} V+\partial_{z} W=0 \quad \text { Ec. continuidad en } x, y \text { y } z
$$

Ecuación de Continuidad integrada verticalmente que se usa en el modelo es:

$$
\frac{\partial \eta}{\partial t}+\frac{\partial}{\partial x}[(h+\eta) u]+\frac{\partial}{\partial y}[(h+\eta) v]=0
$$

Para las ecuaciones de momentum y la ecuación de continuidad, la simbología usada se encuentra en la Tabla 1.

Para resolver cada ecuación e integrarla en el tiempo, cada elemento de cambio temporal como: $\partial U / \partial t=$ (ecuación $)$, se transforma a deltas: si $\Delta \mathrm{U}=\mathrm{U}(\mathrm{t}+1)-\mathrm{U}(\mathrm{t})$ :

$$
\mathrm{U}(\mathrm{t}+1)=\mathrm{U}(\mathrm{t})+\Delta \mathrm{t} * \text { (ecuación) } .
$$

Se puede conocer el siguiente dato de corriente, conociendo el actual.

$\mathrm{U}(\mathrm{X}+250)-\mathrm{U}(\mathrm{X}-250) / 500$ calcula la $\mathrm{U}$ media en diferencias finitas centradas en espacio $X$.

$\mathrm{U}(\mathrm{Y}+250)-\mathrm{U}(\mathrm{Y}-250) / 500$ calcula la $\mathrm{U}$ media en diferencias finitas centradas en espacio $\mathrm{Y}$. 
$\mathrm{V}(\mathrm{X}+250)-\mathrm{V}(\mathrm{X}-250) / 500$ calcula la $\mathrm{V}$ media en diferencias finitas centradas en espacio $\mathrm{X}$.

$\mathrm{V}(\mathrm{Y}+250)-\mathrm{V}(\mathrm{Y}-250) / 500$ calcula la $\mathrm{V}$ media en diferencias finitas centradas en espacio $Y$.

El paso de tiempo se calcula más adelante.

El sistema de ecuaciones diferenciales se resuelve por medio del método de diferencias finitas centradas bajo un esquema explícito, por lo tanto el requerimiento de estabilidad se sustenta en el criterio CFL (Courant et al. 1928); para este caso es:

$$
\Delta t \leq \frac{\Delta x}{\sqrt{g H_{\max }}}
$$

donde $\mathrm{H}_{\max }$ es la profundidad máxima en el área modelada, $(\Delta \mathrm{x}=\Delta \mathrm{y})$ son los tamaños de la malla en las direcciones $x \mathrm{e}$ $y$ respectivamente, $\Delta \mathrm{t}$ es el paso del tiempo y $g$ es la aceleración de la gravedad. La condición inicial es el reposo y de ahí evoluciona el campo de corrientes cuando va aumentando el nivel de la marea, que también empieza en cero.

En la simulación se utilizan las condiciones de frontera siguientes: velocidad de la corriente normal a la costa igual a cero $(\mathrm{Vn}=0)$; variación de la velocidad tangencial a la costa nula $(\mathrm{Vt}=0)$; en las fronteras, es decir en la comunicación con el Golfo de California, el gradiente normal de la corriente es nulo (Jiménez-Illescas 1996)

Las condiciones de frontera abiertas se forman por la suma de armónicos de la marea: M2, S2, N2, K2, K101, P1 (Grivel \& Grivel 1991).

$$
Z(x, y, t)=A(x, y) \cdot \sin (\sigma t+f a(x, y))
$$

donde:

$A$ es la amplitud,

$\sigma$ es la frecuencia

$f a$ es la fase

Se forma una función como serie de tiempo, con el paso de tiempo de $10 \mathrm{~s}$, y se va proporcionando el dato del nivel de la superficie libre cuando lo va necesitando el modelo, de esta manera la superficie libre en las fronteras abiertas se mueve igual que en un ciclo típico de la marea en las bocas del sistema, una vez cada paso de tiempo $\Delta \mathrm{t}$.

Se construyó un modelo digital de profundidades que tiene una malla de 250 x $250 \mathrm{~m}$ (suficiente para resolver los canales que tienen varios kilómetros de ancho en las bocas. La batimetría fue registrada con un sonar digital con GPS, directamente desde una panga en el Sistema Lagunar Navachiste, grabando en memoria, registros que posteriormente fueron descargados a una PC. La profundidad máxima del área modelada fue de $12 \mathrm{~m}$, con un paso de tiempo de 20,65 s para satisfacer el requerimiento de estabilidad CFL (Fig. 2). Los datos de descargan directamente a una PC mediante el software MapSource (Garmin Ltd.), se depuran y se convierten a un archivo $\mathrm{X}, \mathrm{Y}, \mathrm{Z}$, se introducen a un sistema de interpolación Global Mapper y se obtiene el modelo digital de profundidades, que es la malla de integración del modelo.

Se usó viento de velocidad uniforme de $5,0 \mathrm{~m} \mathrm{~s}^{-1}$ del noroeste (Jiménez-Illescas 1996). Se utilizó un valor de $10^{-3}$ para el coeficiente de fricción en el fondo y un coeficiente horizontal de viscosidad de $5 \times 10^{-4} \mathrm{~m}^{2} \mathrm{~s}^{-1}$.

La modelación da inicio con un valor de cero para las velocidades y elevaciones en los puntos interiores de la malla (inicia en reposo). Cada período es idéntico al siguiente. La solución numérica se consideró convergente cuando del segundo al tercer período de la marea se determinó una diferencia máxima de $2 \%$ en la magnitud de las velocidades.

El modelo aplicado es un modelo en diferencias finitas, explícito; las diferencias están centradas en espacio y adelantadas en tiempo.

\section{Trabajo de Campo}

En el presente trabajo se analizaron datos oceanográficos disponibles del área de estudio, los cuales fueron obtenidos con trazadores lagrangianos con GPS integrado, durante 2 campañas realizadas desde el 20 al 21 de marzo y desde el 20 al 22 de octubre 2013, a bordo de embarcaciones menores. Las mediciones lagrangianas se realizaron en 3 bocas del sistema: Boca de Ajoro, Boca Vasiquilla y Boca La Bocanita, conectadas con el Golfo de California. Se midieron las corrientes con trazadores lagrangianas, grabando la posición cada minuto del movimiento de la masa de agua del sistema lagunar, durante marzo y octubre 2013. Posteriormente se calculó la trayectoria, velocidades, rumbos de la masa de agua y la oscilación de la marea en el sistema Lagunar Navachiste Fig (3).

\section{Resultados}

El modelo hidrodinámico numérico, permitió establecer las características de las corrientes en las lagunas estudiadas.

La simulación de la marea en Boca Ajoro y corrientes de flujo fueron del orden de 1,4 $\mathrm{m} \mathrm{s}^{-1}$ y corrientes de reflujo en el orden de 1,5 $\mathrm{m} \mathrm{s}^{-1}$ en Boca Ajoro. En Vasiquilla la rapidez fue de 1,3 $\mathrm{m} \mathrm{s}^{-1}$ y en La Bocanita alcanzan 1,2 $\mathrm{m} \mathrm{s}^{-1}$ en el reflujo mayor en marea de sicigia. Se observa un retraso en la presentación del máximo de unos 6 a 7 min entre bocas, iniciando por La Bocanita, Vasiquilla y Ajoro (Fig. 4). 


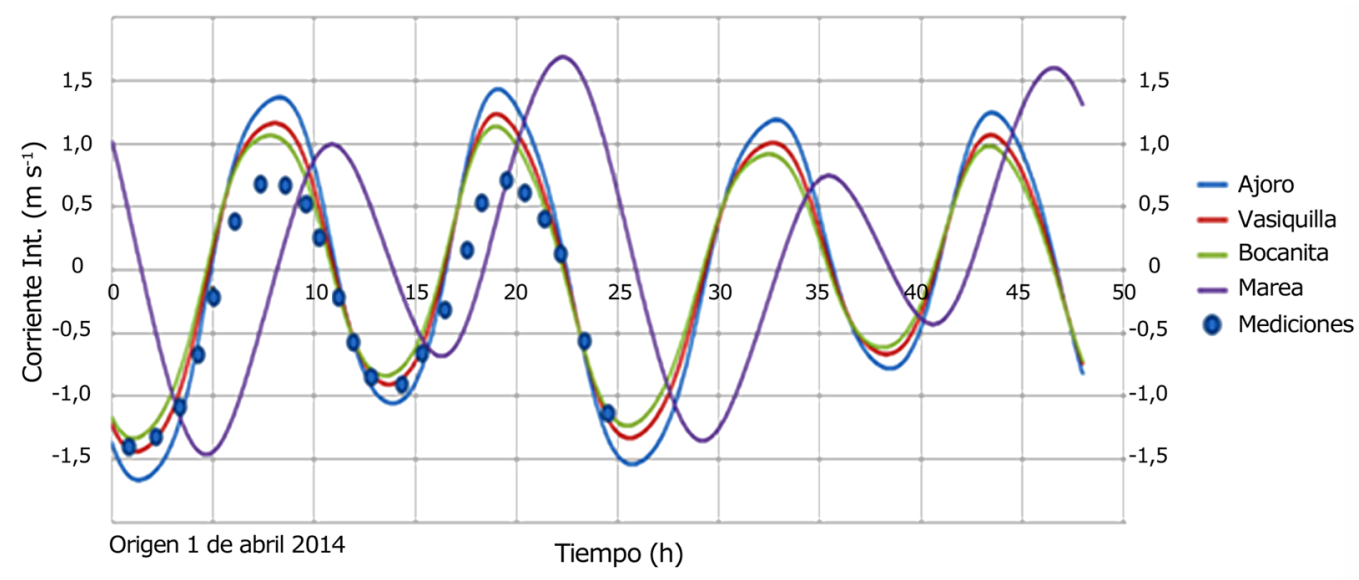

Figura 4. Simulación diaria de marea y corrientes de flujo y reflujo en Boca Ajoro, Vasiquilla y La Bocanita en marea de sicigia / Daily simulation of tide and currents of flow and reflux in the Ajoro, Vasiquilla mouth and the La Bocanita in syzygy tide

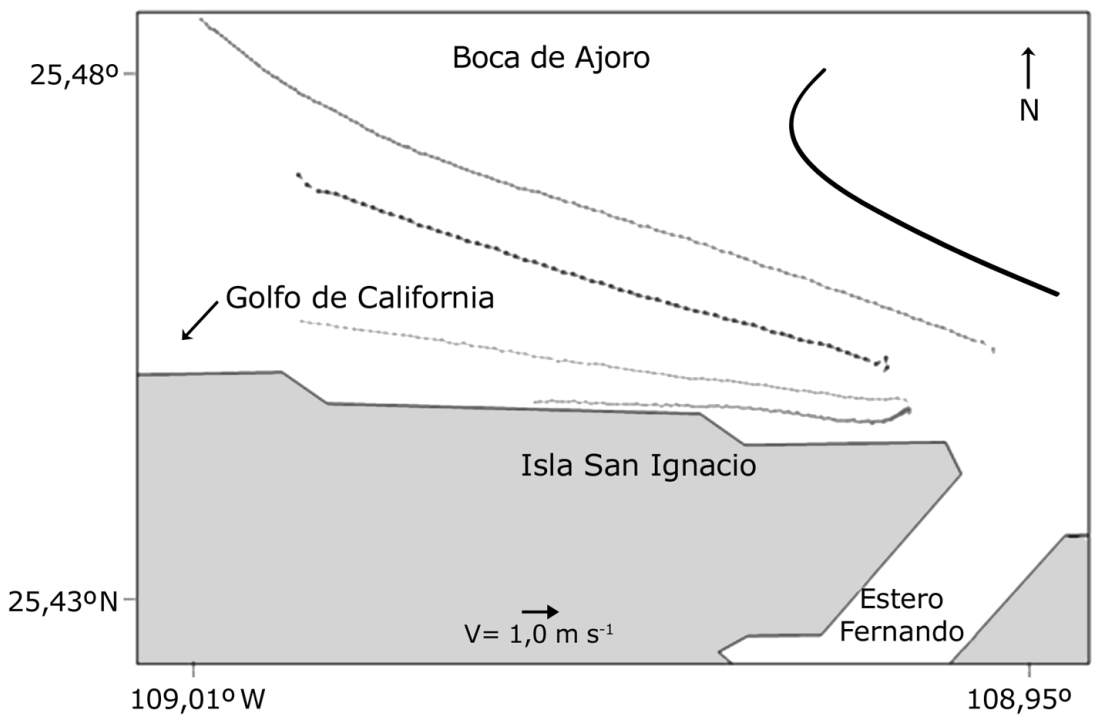

Figura 5. Campo vectorial de corrientes durante el 21 y 22 de marzo 2013 en la Boca de Ajoro del Sistema Lagunar Navachiste / Vector field of currents during the 21 and 22 March 2013 in the Ajoro mouth of the Navachiste Lagoon System

Los trazadores lagrangianos lanzados el 21 de marzo 2013 frente a el Estero Fernando muestran la marea en reflujo y se dirigieron hacía el canal en la Boca de Ajoro en la frontera con el GC, con una rapidez promedio de $0,40 \mathrm{~m} \mathrm{~s}^{-1}$, así como una rapidez máxima de $0,66 \mathrm{~m} \mathrm{~s}^{-1}$. El trazador más alejado de la costa de la Isla San Ignacio, en su recorrido se desvía a la derecha y se dirige al interior de la Laguna San Ignacio, debido al efecto de los vientos del noroeste y a la rotación de la Tierra (Fig. 5).
Los trazadores lagrangianos en Boca Vasiquilla durante el flujo de la marea el día 22 de marzo de 2013 se dirigen hacia el interior de la Laguna Navachiste y mostraron una tendencia a converger en la parte profunda de los canales, con una rapidez de $1,3 \mathrm{~m} \mathrm{~s}^{-1}$ al oeste de la Isla Vinorama (Fig. 6a). Mientras que durante el reflujo del día 22 marzo del 2013, el trazador lagrangiano al este de la Isla San Ignacio se desplaza hacia el GC con una rapidez del orden de $0,2 \mathrm{~m} \mathrm{~s}^{-1}$ (Fig. 6b). 

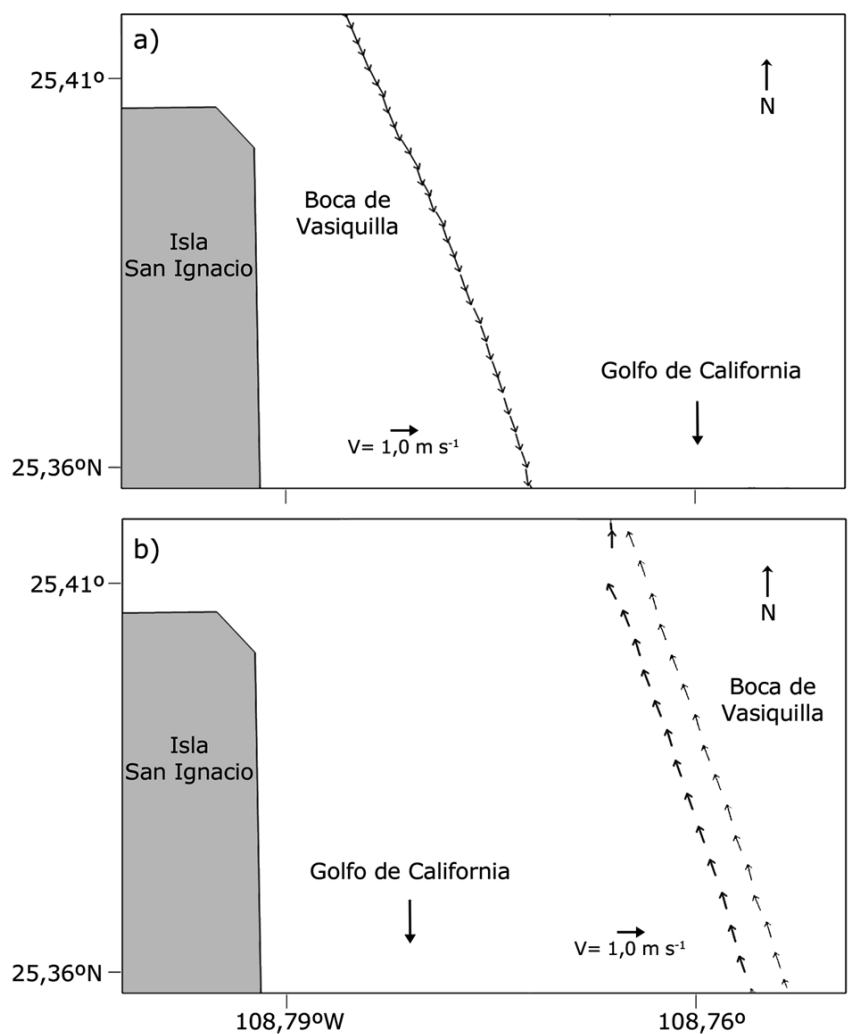

Figura 6. Campos vectoriales de corrientes durante el 21 y 22 de marzo 2013 en la Boca de Vasiquilla del Sistema Lagunar Navachiste durante: a) Reflujo y b) Flujo / Vector fields of currents during the 21 and 22 March 2013 in the Vasiquilla mouth of the Navachiste Lagoon System during: a) Reflux and b) Flow
Durante el 19 de octubre 2013, se presentaron corrientes en dirección al reflujo sobre el canal de la Boca de Ajoro, los mismo patrones observados durante el muestreo de marzo, sin embargo, las velocidades alcanzadas variaron en octubre, donde la mínima fue de alrededor de $0,30 \mathrm{~m} \mathrm{~s}^{-1}$, mientras que la velocidad máxima durante el reflujo alcanzó promedios de $0,90 \mathrm{~m} \mathrm{~s}^{-1}$ (Fig. 7).

En el registro de las trayectorias lagrangianas del 21 de octubre 2013 en Boca Vasiquilla se registraron velocidades de $0,90 \mathrm{~m} \mathrm{~s}^{-1}$ frente a la Isla San Ignacio, mientras que frente a la Isla Vinorama las velocidades registraron superaron $1,0 \mathrm{~m} \mathrm{~s}^{-1}$ en la zona del canal (Fig. 8).

En la Boca La Bocanita el 21 de octubre de 2013, las trayectorias lagrangianas se dirigieron hacia la parte profunda del canal y posteriormente a la Playa Las Glorias con una rapidez promedio de $0,54 \mathrm{~m} \mathrm{~s}^{-1}$. En la zona protegida de la playa (espigón) la rapidez se incrementó de $0,75 \mathrm{~m} \mathrm{~s}^{-1}$ a $1,40 \mathrm{~m} \mathrm{~s}^{-1}$ (Fig. 9).

La corriente máxima registrada en la Boca de Vasiquilla, tuvo una magnitud de $0,90 \mathrm{~m} \mathrm{~s}^{-1}$ y en las costas de la Isla San Ignacio e Isla Vinorama las velocidades máximas fueron de 1,0 $\mathrm{m} \mathrm{s}^{-1}$, mientras que las corrientes modeladas tienen una velocidad entre $0,5 \mathrm{~m} \mathrm{~s}^{-1}$ y $1,2 \mathrm{~m} \mathrm{~s}^{-1}$ (Tabla 2).

Las corrientes registradas en La Bocanita durante las campañas, fueron impulsadas por la marea, sin embargo, dicha velocidad se incrementó al acercase al espigón ya que se reduce la sección transversal haciendo que aumente la velocidad hasta $1,4 \mathrm{~m} \mathrm{~s}^{-1}$ (Tabla 2).

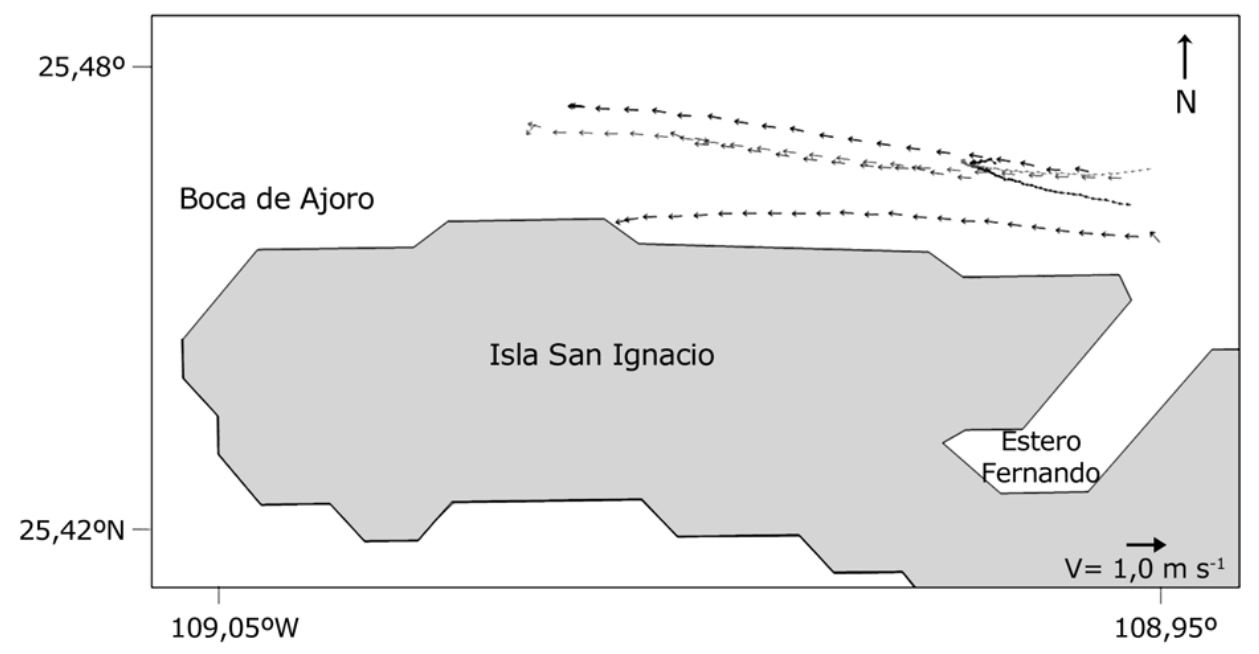

Figura 7. Campo vectorial de corrientes durante el 19 de octubre 2013 en la Boca de Ajoro del Sistema Lagunar Navachiste / Vector field of currents during October 19, 2013 in the Ajoro mouth of the Navachiste Lagoon System 


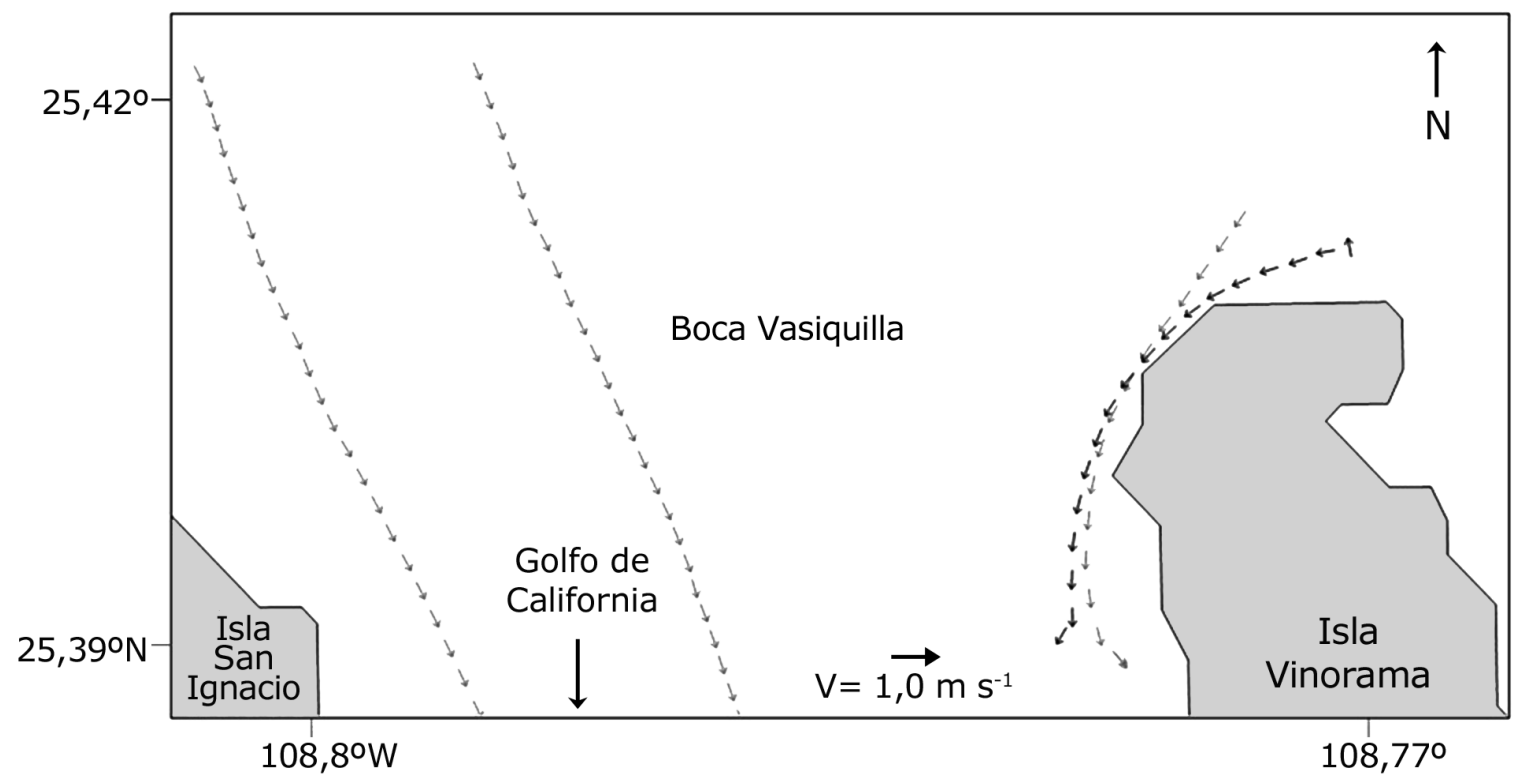

Figura 8. Campo vectorial de corrientes durante el 20 de octubre 2013 en la Boca Vasiquilla del Sistema Lagunar Navachiste / Vector field of currents during October 20, 2013 in the Vasiquilla mouth of the Navachiste Lagoon System

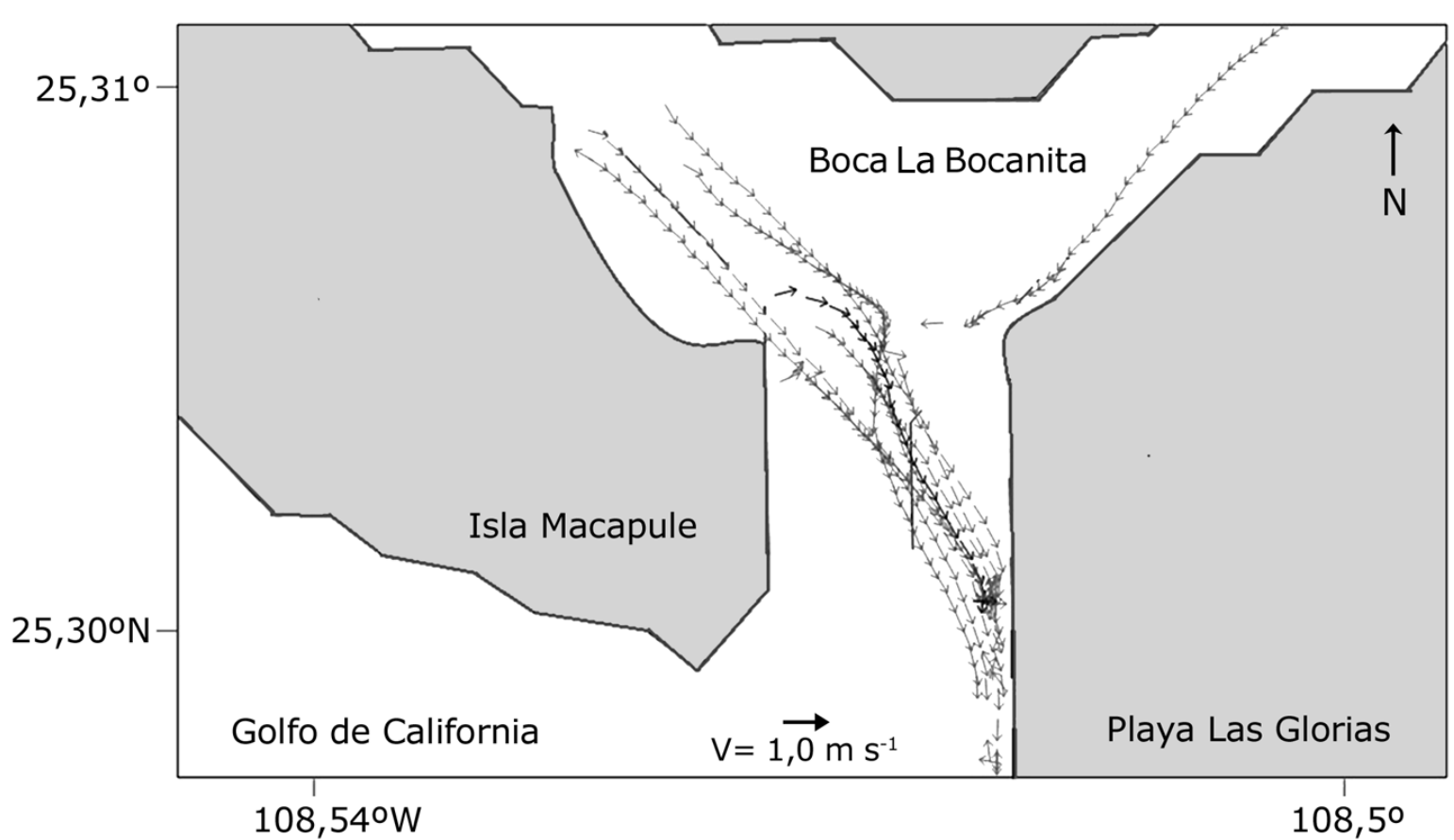

Figura 9. Campo vectorial de corrientes durante el 21 de octubre 2013 en La Bocanita del Sistema Lagunar Navachiste / Vector field of currents during the October 21, 2013 in the La Bocanita of the Navachiste Lagoon System 
Tabla 2. Valores estadísticos (en $\mathrm{m} \mathrm{s}^{-1}$ ) de la velocidad registrada y la modelada del 21 de marzo (20 de octubre) del 2013, en Boca Ajoro, del 21 de marzo (21 de octubre) del 2013, en Boca Vasiquilla y 22 de octubre de 2013, Boca La Bocanita, Sistema Lagunar Navachiste, Sinaloa, México / Statistics values (in m s${ }^{-1}$ ) of the speed recorded and the modeled on 21 March (October 20) from 2013, in Ajoro mouth, of 21 March (October 21) in 2013, in the Vasiquilla mouth and 22 October 2013, La Bocanita mouth, Navachiste Lagoon System, Sinaloa, Mexico

\begin{tabular}{lccccccccc}
\hline & \multicolumn{2}{c}{ Boca Ajoro } & & \multicolumn{2}{c}{ Boca Vasiquilla } & & \multicolumn{2}{c}{ Boca La Bocanita } \\
\cline { 2 - 3 } Máximo & Registrada & Modelada & & Registrada & Modelada & & Registrada & Modelada \\
Minino & 0,703 & 0,632 & & 1,242 & 1,201 & & 0,541 & 0,561 \\
Intervalo & 0,452 & 0,381 & & 0,423 & 0,352 & & 0,351 & 0,282 \\
Media & 0,251 & 0,251 & & 0,819 & 0,849 & & 0.190 & 0.279 \\
Desv. Estándar & 0.578 & 0,565 & & 0,877 & 0,852 & & 0.456 & 0,444 \\
Coef. Correlación & 0.087 & 0,084 & & 0,237 & 0,336 & & 0,071 & 0,078 \\
\hline
\end{tabular}
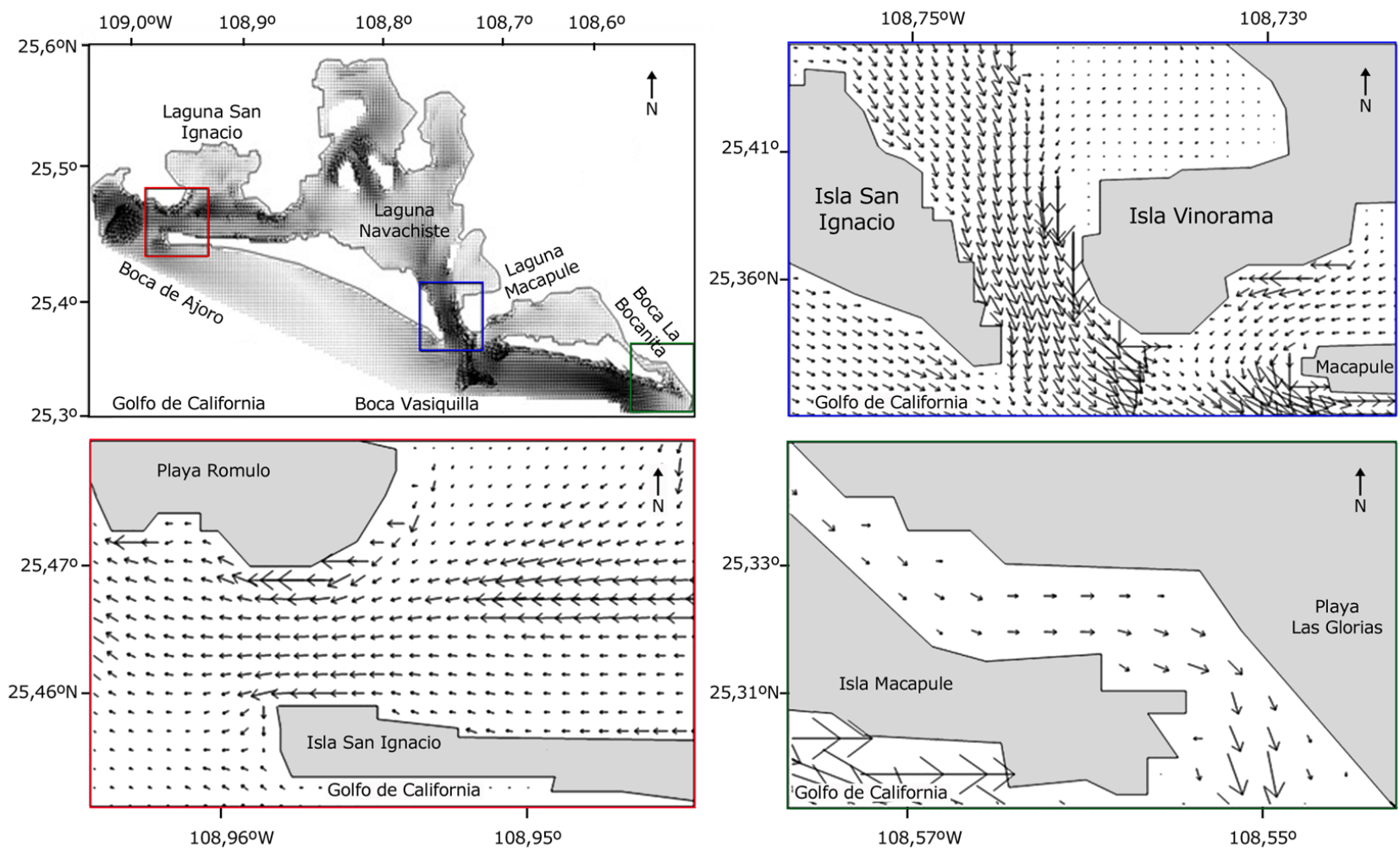

Figura 10. Distribución vectorial de corrientes durante el reflujo de la marea, y el forzamiento de viento uniforme de $5 \mathrm{~m} \mathrm{~s}^{-1}$ del noroeste / Currents distribution vector during reflux of the tide and wind forcing uniform $5 \mathrm{~m} \mathrm{~s}^{-1}$ from the Northwest

La modelación numérica de las corrientes inducidas por la marea y por un viento uniforme del noroeste de $5 \mathrm{~m} \mathrm{~s}^{-1}$ para toda la malla, generaron las mayores velocidades, en el cuarto octavo del ciclo de la marea $\left(0,63 \mathrm{~m} \mathrm{~s}^{-1}\right)$, en Boca de Ajoro durante el reflujo, mostrando una corriente paralela a la línea costa. La velocidad de la corriente fue más intensa, en la zona de calanes de Boca Ajoro adyacente a la costa continental. En los canales de Boca de Ajoro y Boca Vasiquilla, se obtuvieron velocidades que alcanzaron $1,2 \mathrm{~m} \mathrm{~s}^{-1}$ durante el reflujo. Las líneas de corriente siguen los contornos batimétricos en el Sistema Lagunar, con velocidades máximas en la zona de los canales y en las bocas. 

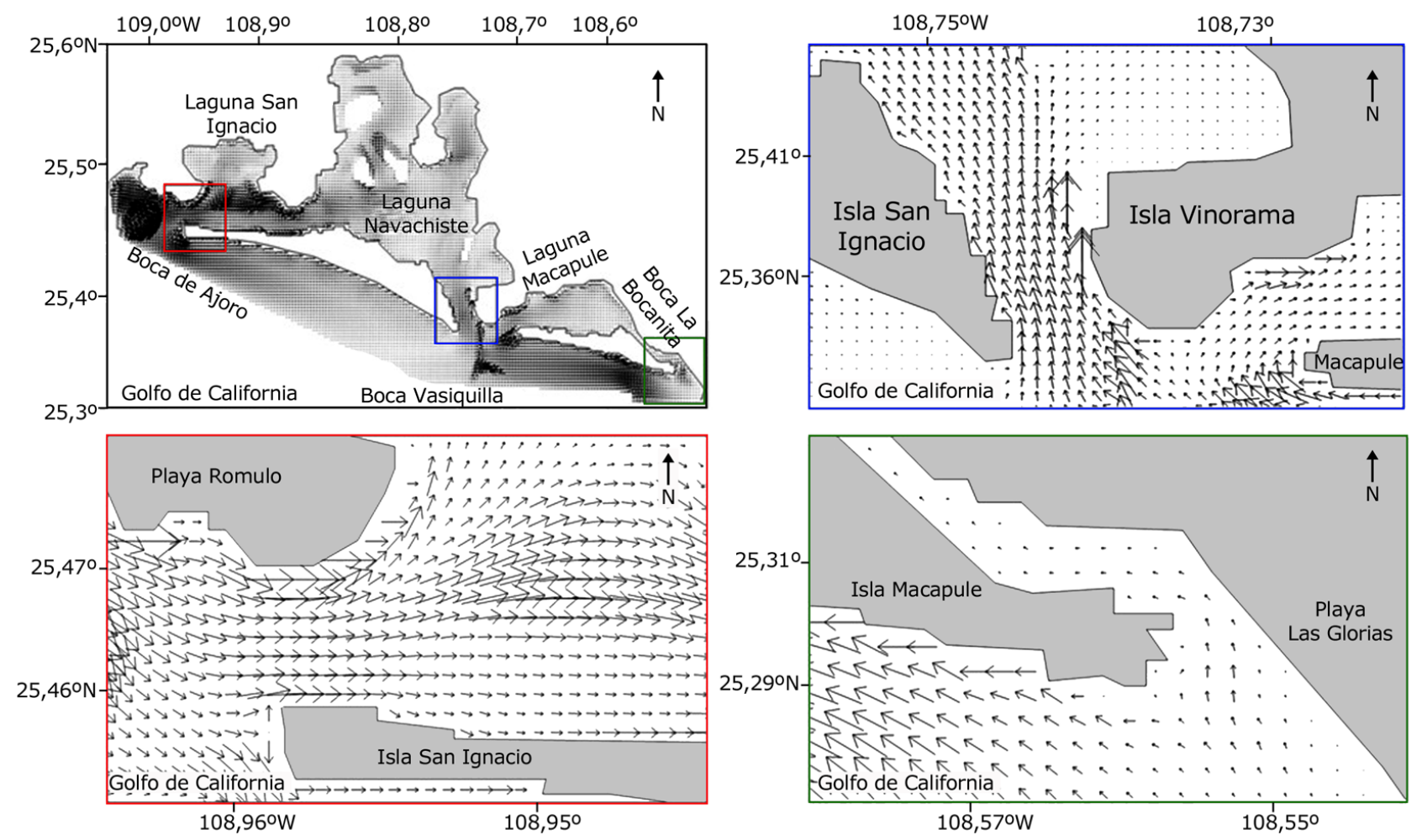

Figura 11. Distribución vectorial de corrientes durante el flujo de la marea, y el forzamiento del viento uniforme de $5 \mathrm{~m} \mathrm{~s}^{-1}$ del noroeste / Current distribution vector during tidal flow and wind forcing uniform $5 \mathrm{~m} \mathrm{~s}^{-1}$ from the Northwest

En la parte sureste del sistema, en Boca La Bocanita durante el reflujo en el cuarto octavo simulado del ciclo de la marea se aprecian las máximas velocidades en la comunicación con el GC, generando en la línea de costa una importante erosión en la parte oeste de Playa Las Glorias, Guasave, Sinaloa (Fig. 10).

Las velocidades máximas de flujo en el modelo numérico corresponden al tercer octavo de la marea, con magnitudes del orden de $\left(0,63 \mathrm{~m} \mathrm{~s}^{-1}\right)$, Con las velocidades máximas frente a la costa continental en Boca de Ajoro, mientras que en Boca de Vasiquilla las velocidades con mayor rapidez se simularon frente a la costa de Laguna Macapule y en Boca La Bocanita la simulación generó las velocidades más rápidas frente a la Playa Las Glorias. Los patrones de circulación el sistema lagunar durante el flujo giran en sentido contrario a las manecillas del reloj a la altura de la región de islas en la laguna de Navachiste, estableciéndose una condición de flujo permanente a través de las Bocas de Ajoro y Vasiquilla, produciendo dos zonas de convergencia. Mientras que en La Bocanita, los vectores de mayor magnitud se ven orientados sobre la zonas de mayor profundidad y paralelos al espigón (Fig. 11).

\section{Discusión}

La distribución vectorial de corrientes que genera el modelo numérico, es forzada por la marea y por el viento medio sinóptico de intensidad constante, proveniente del noroeste, condición semejante a la que se presentó durante las mediciones lagrangianas en Boca Ajoro y Boca de Vasiquilla durante el flujo en marzo 2013 y durante el reflujo en octubre 2013 en las 3 bocas del Sistema Lagunar Navachiste.

De acuerdo con los resultados obtenidos, la hidrodinámica del Sistema Lagunar Navachiste, es similar a la hidrodinámica de las lagunas costeras someras del Pacífico y el Mar de Cortés, de la República Mexicana, presentándose las máximas velocidades en canales y bocas, donde las intensidades de las corrientes alcanzan velocidades superiores a $1 \mathrm{~m} \mathrm{~s}^{-1}$. Estos patrones se han reportado previamente en otros sistemas lagunares de la región (Cárdenas-Gámez 2007). Por otra parte, es importante destacar que las máximas velocidades se presentan durante los periodos de reflujo de la marea, razón por lo que prevalecen abiertas las bocas del sistema y sin azolvarse, siendo ésta una característica muy importante de las lagunas costeras en la región. Un comportamiento hidrodinámico similar se presenta en los sistemas Topolobampo-Santa María 
en Ahome, El Colorado en Ahome y Santa María en La Reforma (Escobedo-Urías 1997, Cárdenas-Gámez 2007).

La hidrodinámica dentro del Sistema Lagunar Navachiste, está influida principalmente por 2 factores: las mareas y el efecto del viento, características representativas de las lagunas de Pacífico mexicano. Un comportamiento similar fue reportado por Jiménez-Illescas (1996) para la Bahía de La Paz, ObesoNieblas et al. (1996) para Bahía Concepción, Obeso-Nieblas et al. (1999) para el Sistema Lagunar Bahía MagdalenaAlmejas. De La Lanza \& Flores-Verdugo (2002) y ObesoNieblas et al. (2012) para Bahía Concepción y Obeso-Nieblas et al. (2002), para la Bahía de La Paz, BCS.

En un estudio similar Gaviño \& Fernández (1987), utilizaron un modelo Hidrodinámico barotrópico bidimensional para predecir las corrientes inducidas por la marea y viento, el modelo se adaptó, estructuró y resolvió de acuerdo a las condiciones propias de la albufera Barra de Navidad y a las ecuaciones de momento y continuidad, ya que simula el movimiento de partículas de un flujo con la misma densidad. Dicho modelo también fue utilizado por Ramírez-León et al. (2013) quienes obtuvieron la simulación de la descarga de la Planta Termo Nuclear de Laguna Verde, en el Estado de Veracruz, con el objetivo de conocer el comportamiento de las plumas térmicas en las aguas costeras.

Los resultados obtenidos de la simulación hidrodinámica en el Sistema Lagunar Navachiste, coinciden en $87 \%$ con los registros obtenidos en el campo por los instrumentos de medición en Boca de Ajoro y Boca Vasiquilla en marzo 2013 y en las 3 comunicaciones con el Golfo de California en octubre 2013 (Tabla 2).

Las condiciones registradas en el sistema Navachiste, durante los muestreos de marzo y octubre 2013, fueron producto de los principales mecanismos de generación hidrodinámica como el componente de mareas y los vientos del noroeste, dichas características son similares a las reportadas por EscobedoUrías (1997).

Analizando el campo vectorial de las corrientes en el sistema lagunar se puede observar una circulación de Bahía San Ignacio hacia Bahía Macapule durante el flujo, este proceso se revierte durante el reflujo. Esto significa que el agua entra por la boca de Ajoro y fluye hasta la parte sur de Bahía Navachiste. Esto difiere ligeramente de los resultados de (Sanay-González et al. 2006), obtenidos en el Sistema Lagunar Chacahua-Pasotiria en el estado de Oaxaca, quienes presentan resultados de un modelo numérico no-lineal de circulación, quienes consideran en sus estudios que la velocidad de la corriente de entrada es mayor que la de salida, esto indica la asimetría en los flujos de entrada y salida, lo cual puede ocasionar un transporte neto de material en suspensión y/o carga de fondo que azolva la laguna oaxaqueña.

El Sistema Lagunar Navachiste es un cuerpo de agua semicerrado, su circulación es similar a la de otros sistema lagunares del Pacífico y Golfo de California, como fue observado durante los años de estudio. Una situación similar fue reportada en un sistema lagunar somero por ObesoNieblas et al. (1999), quienes describen la simulación numéricamente de los componentes de marea en el Sistema Lagunar Bahía de Magdalena-Almejas, B.C.S., mediante un modelo hidrodinámico barotrópico, los resultados del modelo se comparan, cuantitativa y cualitativamente, con mediciones de campo de variaciones del nivel del mar y velocidad de corriente, obteniendo velocidades máximas, registradas y modeladas durante el reflujo de la marea.

Los resultados de los campos vectoriales de corrientes del modelo hidrodinámico muestran las velocidades máximas de flujo en el tercer octavo de la marea en las bocas del sistema y en la zona de los canales, mientras que durante el reflujo las velocidades máximas se obtuvieron en el cuarto octavo de la marea, también en las bocas del sistema y en la zona de canales.

Finalmente, se concluye, que los cuerpos de deriva tuvieron una trayectoria hacia la parte más profunda de los canales durante el reflujo, con velocidades más intensas que en el flujo, lo que permite mantener las bocas abiertas del sistema lagunar.

Los campos vectoriales generados por la simulación numérica en el sistema lagunar, mostraron un comportamiento hidrodinámico similar a las trayectorias trazadas por los cuerpos de deriva durante las campañas de muestreo.

\section{Agradecimientos}

A los proyectos SIP-20150585 del Instituto Politécnico Nacional (IPN). El autor tiene becas CONACYT y BEIFI del IPN. LEC tiene becas COFAA y EDI. Al Lic. Renato Leal Moreno y al Lic. José Gaspar Ángulo Machado por el apoyo de la obtención de datos utilizados en este trabajo.

\section{LiTERATURA CITADA}

Bustillos-Guzmán J, H Claustre \& JC Marty. 1995. Specific phytoplankton signatures and their relationship to hydrographic conditions in the coastal North-western Mediterranean Sea. Marine Ecology Progress Series 124: 247-258.

Cárdenas-Gámez S. 2007. Modelación hidrodinámica de la Laguna Costera El Colorado, Ahome, Sinaloa. Tesis de Maestría, Centro Interdisciplinario de Investigación para el Desarrollo Integral Regional, Instituto Politécnico Nacional, Guasave, 94 pp. 
Cifuentes J, R Rodríguez \& A Zarur. 1972. Panorama general de la contaminación de las aguas en México. En: Ruivo M (ed). Marine pollution and sea life, pp. 100-106. FAO, Fishing News Books, Roma.

Courant R, K Friedrichs \& H Lewy. 1928. Uber die partiellen differenzeng leichungen der matematischen physic. Mathematische Annalen 100: 32-74.

De la Lanza-Espino G \& C Cáceres-Martínez. 1994. Lagunas costeras y el litoral mexicano, 525 pp. Universidad Autónoma de Baja California Sur, La Paz.

De la Lanza-Espino G \& F Flores-Verdugo. 2002. Oceanografía de Sinaloa. En: Cifuentes JL \& J Gaxiola (eds). Atlas de la biodiversidad de Sinaloa, pp. 365-379. El Colegio de Sinaloa, Culiacán.

Douglas M, R Maddox, K Howard \& S Reyes. 1993. The Mexican monsoon. Journal of Climate 6: 1665-1677.

Dworak J. 2005. Modulación de mareas someras en régimen de tipo mixto. Tesis de Doctorado en Ciencias, Centro de Investigaciones Biológicas del Noroeste, S.C., La Paz, 98 pp. <http://cybertesis.cibnor.mx:8080/sdx/cibnor/2005/ dworak_j.pdf $>$

Escobedo-Urías D, M Hernández, N Herrera, A Ulloa \& A Chiquete. 1999. Calidad bacteriológica del sistema lagunar de San Ignacio-Navachiste, Sinaloa. Ciencia y Mar 3: 1727.

Escobedo-Urías D, A Martínez, Á Jiménez, A Ulloa \& A Zavala. 2007. Intercambio de carbono orgánico particulado del sistema lagunar San Ignacio Navachiste, Sinaloa, con el mar adyacente. En: Hernández de la Torre B \& G GaxiolaCastro (eds). Carbono en sistemas acuáticos de México, pp. 171-185. INE-CICESE, México.

Espinosa-Carreón TL \& J Valdez-Holguín. 2007. Variabilidad interanual de clorofila en el Golfo de California. Ecología Aplicada 26(4): 561-583.

Flores-Verdugo F, O Calvario \& M Cárdenas. 1993. Características ambientales de los humedales de Sinaloa y Nayarit. Humedales Costeros de México 2(1): 11-16. Oficina de humedales para las Américas en México, ITESMGuaymas.

García E. 1973. Modificaciones al sistema de clasificación de Koopen, 246 pp. Instituto de Geofísica, Universidad Nacional Autónoma de México, México.

Gaviño R \& E Fernández. 1987. Modelación hidrodinámica numérica de la Albufera Barra de Navidad, Jalisco, 40 pp. Instituto Oceanográfico de Manzanillo, Universidad de Colima, México.

Grivel FP \& FV Grivel. 1991. Tablas de predicción de mareas 1992. Puertos del Océano Pacífico, pp. 113-127. Instituto de Geofísica, UNAM, México.

Jiménez A, M Obeso \& M Alatorre. 1994. Hidrología de la Bahía de La Paz. Oceanología, Unidad de Educación en Ciencia y Tecnología de Mar 1: 115-131.
Jiménez-Illescas AR. 1996. Análisis de procesos barotrópicos y baroclínicos en la Bahía de La Paz, BCS. Tesis Doctoral, Universidad Nacional Autónoma de México, México DF, 212 pp.

Jiménez-Illescas AR, M Obeso-Nieblas \& D Salas. 1997. Oceanografía física de la Bahía de La Paz, BCS. En: Urbán RJ \& M Ramírez (eds). La Bahía de La Paz, Investigaciones y conservación, pp. 31-41. Universidad Autónoma de Baja California Sur, Centro Interdisciplinario de Ciencias Marinas, Scripp Institute Oceanographic, La Paz.

Magaña-Álvarez M. 2004. Distribución de nutrientes y su efecto en el nivel trófico de la laguna de Macapule, Sinaloa. Tesis de Maestría, Centro Interdisciplinario de Investigación para el Desarrollo Integral Regional, Instituto Politécnico Nacional, Guasave, 105 pp.

Marinone S \& M Lavín. 1997. Mares y corrientes residuales en el Golfo de California. En: Lavin MF (ed). Contribuciones a la oceanografía física en México. Monografía 3: 113-139. Unión Geofísica Mexicana, Ensenada.

Martínez-López A, D Escobedo, A Ulloa \& C Band. 2006. Bloom of Chattonella subsalsa in a eutrophic coastal lagoon in the Gulf of California. Harmful Algae News 31(1): 4-5.

Martínez-López A, D Escobedo, A Reyes \& M Hernández. 2007. Phytoplankton response to nutrients runoff in a Lagoon System in the Gulf of California. Hidrobiológica 17(2): 101112.

Millán-Nuñez E, F Ortiz-Cortez \& S Álvarez-Borrego. 1981. Variabilidad temporal y espacial de nutrientes y fitoplancton en una laguna costera a finales de verano. Ciencias Marinas 7(1): 103-128.

Molina-Cruz A. 1984. Evaluación oceanográfica de la Boca del Golfo de California. Anales del Instituto de Ciencias del Mar y Limnología, Universidad Nacional Autónoma de México 13(2): 95-120.

Obeso-Nieblas M, A Jiménez \& S Troyo. 1993. Modelación de la marea en la Bahía de La Paz, B.C.S. Investigaciones Marinas CICIMAR 8(1): 13-22.

Obeso-Nieblas M, MA Alatorre-Mendieta \& AR JiménezIllescas. 1996. Modelación de la marea en Bahía Concepción, B.C.S., México. Oceánides 11(1): 1-8.

Obeso-Nieblas M. 1987. Propagación de la constituyente M2 de la marea en la Bahía de La Paz, B. C. S., México, mediante un modelo hidrodinámico numérico. Tesis de Maestría, Centro Interdisciplinario de Ciencias Marinas, Instituto Politécnico Nacional, La Paz, 123 pp.

Obeso-Nieblas M, A Jiménez \& J Gaviño. 1999. Modelación de la marea en el sistema lagunar Bahía Magdalena, B.C.S., México. Oceánides 14(2): 79-88.

Obeso-Nieblas M, JH Gaviño-Rodriguez, AR JiménezIllescas \& B Shirasago-German. 2002. Simulación numérica de la circulación por marea y viento del noroeste y sur en la Bahía de La Paz, B.C.S. Oceánides 17(1): 1-12. 
Obeso-Nieblas M, J Gaviño-Rodríguez \& H ObesoHuerta. 2012. Variabilidad espacial y estacional de temperatura, salinidad, y densidad en Bahía Concepción, Golfo de California, México. Revista de Biología Marina y Oceanografía 47(3): 489-502.

Poot-Delgado C. 2006. Estructura de la comunidad fitoplanctónica con énfasis en las especies tóxicas y/o nocivas de la laguna de Macapule, Sinaloa. Tesis de Maestría, Centro Interdisciplinario de Ciencias Marinas, Instituto Politécnico Nacional, La Paz, 142 pp.

Ramírez-León H, C Couder, I Herrera \& H Barrios. 2013. Modelación numérica de la descarga térmica de la Central Nucleoeléctrica Laguna Verde. Revista Internacional Métodos Numéricos para Cálculo y Diseño Ingeniería 29(2): 114-121.

Roden G. 1958. Oceanographic and meteorological aspects of the Gulf of California. Pacific Science 12: 21-45.
Roden G. 1972. Thermohaline structure and baroclinic flow across the Gulf of California entrance and in the Revilla Gigedo Islands region. Journal of Physical Oceanography 22: $177-183$.

Salas D. 1996. El intercambio de agua sobre umbrales del Golfo de California. Tesis de Maestría, Centro de Investigación Científica y de Educación Superior de Ensenada, Baja California, México, Ensenada, 65 pp.

Sanay-González R, M Monreal \& D Salas. 2006. Simulación de la circulación en el sistema lagunar Chacahua-Pastoría, Oaxaca, México. Ciencia y Mar 10(29): 3-16.

Zayas-Esquer MM. 2010. Efectos en la Playa Las Glorias causados por la construcción del espigón al modificar el transporte litoral. Tesis de Maestría, Centro Interdisciplinario de Investigación para el Desarrollo Integral Regional, Instituto Politécnico Nacional, Guasave, 126 pp. 